\title{
Three-Dimensional Isotropic Fat-Suppressed Proton Density- Weighted MRI at 3 Tesla Using a T/R-Coil Can Replace Multiple Plane Two-Dimensional Sequences in Knee Imaging
}

\section{Drei-dimensionale isotrope Protonen-gewichtete fettunterdrückte MRT bei 3 Tesla als Substitut multiplanarer zwei-dimensionaler Knie Sequenzen}

\author{
Authors

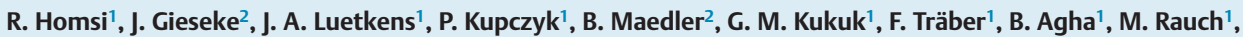 \\ N. Rajakaruna' ${ }^{1}$ W. Willinek ${ }^{3}$, H. H. Schild ${ }^{1}$, D. R. Hadizadeh ${ }^{1}$ \\ Affiliations \\ Department of Radiology, University of Bonn, Germany \\ Philips Healthcare, Hamburg, Germany \\ 3 Center for Radiology, Neuroradiology, Sonography and Nuclear Medicine, Krankenhaus der Barmherzigen Brueder Trier, \\ Germany
}

Key words
\%nee
\& $\mathrm{D}$
\& MRI
multiplanar reformation
proton density-weighted

received $\quad 23.10 .2015$ accepted 26.6.2016

\section{Bibliography}

Dol http://dx.doi.org/

10.1055/s-0042-111826

Published online: 24.8.2016

Fortschr Röntgenstr 2016; 188:

949-956 @ Georg Thieme

Verlag KG Stuttgart · New York .

ISSN 1438-9029

\section{Correspondence \\ Herr Dr. Dariusch Reza \\ Hadizadeh}

Radiologische Klinik, Universitätsklinikum Bonn Sigmund-Freud Str.25

53127 Bonn

Germany

Tel.: ++ 49/228/28715960

Fax: ++ 49/228/28715598

dariusch.hadizadeh@ukb.uni-

bonn.de

\section{Zusammenfassung \\ $\nabla$}

Ziel: Klärung der Frage, ob eine dreidimensionale (3D) isotrope Protonen-gewichteten fettunterdrückte (PDwFS) Sequenz vom Knie mehrere zweidimensionale (2D) Sequenzen ersetzen kann. Material und Methoden: 52 Patienten (26 Männer, 26 Frauen, mittleres Alter $41,9 \pm 14,5 \mathrm{Jahre}$ ) erhielten eine magnetresonanztomografische Bildgebung (MRT) vom Knie unter Nutzung einer $\mathrm{T} / \mathrm{R}$ Spule an einem 3-Tesla-(T)-Magneten. Das Protokoll enthielt 3 Ebenen 2D-PDwFS (Gesamtaufnahmedauer (AD), 6:40 min; Voxelgröße [V], $\left.0,40-0,63 \times 0,44-0,89 \times 3 \mathrm{~mm}^{3}\right)$ und eine 3DPDwFS (AD, 6:31 min; V, 0,63×0,68×0,63 $\mathrm{mm}^{3}$ ). Beurteilt wurden die Homogenität der Fettunterdrückung, Artefakte, und die Bildschärfe anhand einer 5-Punkteskala (5[Exzellent] - 1[nicht-diagnostisch]). Die Summe der Parameter diente als ein Maß für die Gesamtbildqualität (GBQ). Zusätzlich wurden Kontrastverhältnisse (KR) für den Meniskus (MEN), das vordere (ACL) und das hintere Kreuzband (PCL) im Vergleich zum Musculus popliteus berechnet. Von den 52 Patienten erfolgte bei 13 Patienten aus klinischer Indikation eine KnieAthroskopie. Bei diesen Patienten untersuchten zwei unabhängige Radiologen die 3D- und 2D-Aufnahmen auf das Vorhandensein von Läsionen des Meniskus, der Bänder und des Knorpels. Hierauf basierend wurden die Sensitivität und Spezifizität berechnet.

Ergebnisse: Die KR waren für ACL, PCL und MEN in der 3D-PDwFS höher als bei der 2D-PDwFS ( $p<0,01$ für $\mathrm{ACL}$ und $\mathrm{PCL} ; \mathrm{p}=0.07$ für MEN). Verglichen mit den 2D-Bildern, wurde die GBQ aufgrund von weniger Artefakten und einer homogeneren Fettunterdrückung $(p<0,01)$ in den 3D-PDwFS Bilder höher bewertet, trotz einer geringeren Bildschärfe $(p<0,01)$. Sensitivität und Spezifizität für die Diagnose einer Knieläsion waren für 3D- und 2D-PDwFS ähnlich.

\section{Abstract \\ $\nabla$}

Purpose: To evaluate whether a $3 \mathrm{D}$ proton density-weighted fat-suppressed sequence (PDwFS) of the knee is able to replace multiplanar 2D-PDwFS. Materials and Methods: 52 patients (26 men, mean age: $41.9 \pm 14.5$ years) underwent magnetic resonance imaging (MRI) of the knee at 3.0 Tesla using a T/R-coil. The imaging protocol included 3 planes of 2D-PDwFS (acquisition time (AT): 6:40 min; voxel sizes: $0.40-0.63 \times 0.44-0.89 \times 3 \mathrm{~mm}^{3}$ ) and a 3D-PDwFS (AT: 6:31 min; voxel size: $\left.0.63 \times 0.68 \times 0.63 \mathrm{~mm}^{3}\right)$. Homogeneity of fat suppression (HFS), artifacts, and image sharpness (IS) were evaluated on a 5-point scale (5[excellent] - 1[non-diagnostic]). The sum served as a measure for the overall image quality (OIQ). Contrast ratios (CR) compared to popliteal muscle were calculated for the meniscus (MEN), anterior $(\mathrm{ACL})$ and posterior cruciate ligaments (PCL). In 13 patients who underwent arthroscopic knee surgery, two radiologists evaluated the presence of meniscal, ligamental and cartilage lesions to estimate the sensitivity and specificity of lesion detection.

Results: The CR was higher in the ACL, PCL and MEN in 3D- PDwFS compared to 2D-PDwFS ( $p<0.01$ for ACL and PCL; $p=0.07$ for MEN). Compared to $2 \mathrm{D}$ images, the OIQ was rated higher in 3D-PDwFS images $(\mathrm{p}<0.01)$ due to fewer artifacts and HFS despite the lower IS $(\mathrm{p}<0.01)$. The sensitivity and specificity of lesion detection in 3Dand 2D-PDwFS were similar.

Conclusion: Compared to standard multiplanar 2D-PDwFS knee imaging, isotropic high spatial resolution 3D-PDwFS of the knee at 3.0 T can be acquired with high image quality in a reasonable scan time. Multiplanar reformations in arbitrary planes may serve as an additional benefit of 3DPDwFS. 
Schlussfolgerung: Verglichen mit dem Standard der multiplanaren 2D-PDwFS Knie-Bildgebung, können isotrope hochaufgelöste 3D-PDwFS-Aufnahmen bei 3.0 T mit hoher Bildqualität und ähnlicher Aufnahmedauer akquiriert werden. Ein Vorteil der 3DPDwFS besteht in der Möglichkeit multiplanarer Reformationen in beliebigen Ebenen.

Kernaussagen:

- 3D-PDwFS Knie Aufnahmen sind bei hoher Bildqualität möglich

- 3D-PDwFS Knieaufnahmen können in nur einer Messung in akzeptabler Zeit erzielt werden

- Im Gegensatz zur Standardbildgebung ermöglicht die 3D-PDwFS zusätzlich die Rekonstruktion multiplanarer Reformationen
Key Points:

3D-PDwFS of the knee is acquired with high image quality

$>3 \mathrm{D}-\mathrm{PDwFS}$ can be achieved in only one measurement with a reasonable scan time

$>$ 3D-PDwFS with the advantage of multiplanar reformation may replace 2D-PD-weighted knee MRI

Citation Format:

- Homsi R, Gieseke J, Luetkens JA et al. Three-Dimensional Isotropic Fat-Suppressed Proton Density-Weighted MRI at 3 Tesla Using a T/R-Coil Can Replace Multiple Plane Two-Dimensional Sequences in Knee Imaging. Fortschr Röntgenstr 2016; 188: 949-956

\section{Introduction \\ $\nabla$}

Three-dimensional (3 D) volume data sets with isotropic spatial resolution may be beneficial in magnetic resonance imaging (MRI) of various joints and potentially improve image quality and diagnostic efficiency, especially at high field strengths [1 -6].

Currently, multiple 2D sequences in orthogonal slice orientations are required in musculoskeletal MRI in order to fully cover the anatomy and ensure lesion detection. Diagnostic image quality largely depends on ideal planning of imaging planes, which requires the presence of highly trained and dedicated technicians. Imaging may be time-consuming and require additional sequences in specifically angulated orientations to account for lesions that are not well visualized by standard planes. $2 \mathrm{D}$ sequences are acquired with relatively thick sections with consecutive partial volume effects. They are mostly used with imaging gaps to avoid cross talk and scan time prolongation that may lead to limited imaging accuracy particularly of small structures. These limitations may be overcome by isotropic voxels that allow for the reconstruction of multiplanar reformats (MPRs) in arbitrary orientations including curved and oblique planes [3, 7 -9]. $3 \mathrm{D}$ volume data sets with isotropic resolution may be of advantage as they are acquired with thin continuous sections and no interslice gaps. In $3 \mathrm{D}$ sequences pulsation as well as motion artifacts are less pronounced compared to $2 \mathrm{D}$ sequences due to their inherently thicker slabs.

Proton density-weighted sequences with fat suppression (PDwFS) in multiple planes are currently used as the standard sequences for knee MR imaging in many centers. They provide excellent performance with reduced chemical shift artifacts and an extended range of signal intensities in articular cartilage [10 - 13].

The purpose of this study was to quantitatively and qualitatively evaluate the image quality of isotropic high spatial resolution 3DPDwFS-TSE of the knee at 3.0 Tesla (T) using a transmit/receive coil (T/R-coil) and to evaluate whether isotropic 3D-PDwFS may replace multiple $2 \mathrm{D}-\mathrm{PDwFS}$ planes.

\section{Methods \\ $\nabla$}

\section{Patients}

52 patients ( 26 men; mean age: $41.9 \pm 14.5$ years) with suspected knee injury (22 suspected meniscal tears, 18 suspected ligamental tears, 7 unclear knee swellings, and 5 suspected degenerative lesions) were included in a prospective, intra-individual comparative study. The study protocol was approved by the institutional ethics committee and all patients provided informed consent before inclusion in the study. The exclusion criteria were contrain- dications for MRI (e.g., pacemakers, non-MRI-compatible metallic implants). Clinical treatment was not delayed for any patient because of study participation. All patients were asked to report any adverse events and to comment on the overall toleration of the procedure.

\section{MR Imaging}

All scans were performed on a 3.0-Tesla whole-body scanner equipped with a transmit/receive 16-channel knee coil to cover the entire knee (Ingenia; Philips Healthcare, Best, The Netherlands). Patients were examined feet-first in a supine position. The acquisition parameters for the 3D- and 2D-PDwFS sequences are summarized in $\bullet$ Table 1. All sequences were obtained without contrast medium and were performed within the same session. 2D-PDwFS sequences were acquired without the use of parallel imaging due to restrictions because of signal loss at short echo times when using long echo trains. 3-mm-thick transverse, sagittal and coronal MPR planes were generated from 3D-PDwFS in corresponding planes to 2D-PDwFS. $2 \mathrm{D}$ and 3D-PD sequences were combined with a spectral selective attenuation recovery fat suppression technique (SPAIR) with the following parameters: spectral selective fat saturation with an adiabatic inversion pulse duration of $18 \mathrm{~ms}$ and an inversion delay of $85 \mathrm{~ms}$ for optimal bone marrow and muscular fat suppression. The water-fat shift

Table 1 Technical parameters of 2D- and 3D-PDwFS.

Tab. 1 Technische Parameter der 2D- und 3D-PDwFS.

\begin{tabular}{|lll|}
\hline parameter & 2D-PDwFS & 3D-PDwFS \\
\hline TR/TE (ms) & $3387 / 9.2$ & $1300 / 176$ \\
\hline $\begin{array}{l}\text { flip angle } \\
\text { (degrees) }\end{array}$ & 90 & 90 \\
\hline FOV $(\mathrm{mm})$ & 160 & 144 \\
\hline matrix & $160 \times 89$ & $162 \times 160$ \\
\hline $\begin{array}{l}\text { acquired voxel } \\
\text { (mm }{ }^{3} \text { ) }\end{array}$ & $0.40 \times 0.44 \times 3$ & $0.63 \times 0.63 \times 0.63$ \\
\hline slices & 27 & 254 \\
\hline TSE factor & 11 & 63 \\
\hline $\begin{array}{l}\text { acquisition time } \\
\text { (min:s) }\end{array}$ & $3: 04.2$ (sagittal) & $6: 31.3$ \\
& $1: 33.2$ (coronal) & \\
\hline parallel imaging & none & \\
\hline NSA & 1 & $2 \times 2.9$ (transverse) \\
\hline water-fat shift & 2 pixels (220 Hz/pixel) & 1.3 pixels $(340 \mathrm{~Hz} /$ pixel) \\
\hline fat suppression & SPAIR & SPAIR \\
\hline
\end{tabular}

PDwFS: proton density-weighted fat-suppressed sequence; NSA: number of signal averages; SPAIR: spectral selective attenuation recovery fat suppression technique. 
was 2 pixels (220 Hz/pixel) for 2D-PDwFS and 1.3 pixels $(340 \mathrm{~Hz}$ / pixel) for 3D-PDwFS.

\section{Arthroscopic Knee Surgery}

Clinical evaluation of MR imaging was performed by 2 senior radiologists with more than 10 years of experience in musculoskeletal imaging. Based on these findings, 13 patients ( 5 men, 8 women; mean age: $46.4 \pm 17.0$ ) received arthroscopic knee surgery (AKS) by an experienced orthopedic surgeon within $3-56$ days (mean: $28.9 \pm 20.5$ days) of their MRI examination. Documentation of AKS procedures included evaluation of ligamental (anterior and posterior cruciate ligament [ACL, PCL], collateral ligaments) and meniscal (inner and outer meniscus) structures as well as cartilage lesions. The articular surfaces of knee joints were graded using the Outerbridge classification (grade 0 : normal; grade 1: cartilage softening or superficial fissures; grade 2: deepness of cartilage lesions $<50 \%$; grade 3 : deepness of cartilage lesions $>50 \%$; grade 4 : full-thickness cartilage lesion) [14].

\section{Quantitative Image Analysis}

The quantitative analyses consisted of measuring contrast ratios $(\mathrm{CR})$ of $(\mathrm{A})$ the signal of the anterior cruciate ligament (ACL), the posterior cruciate ligament (PCL) and the meniscus (MEN) compared to $(B)$ the signal of the popliteal muscle: $C R=(A-B) /(A+B)$. Polygonal regions of interest (ROI) were placed in the respective tissues, with each area being as large as possible to cover the tissue of interest while avoiding inclusion of confounding structures (e.g. blood vessels). The signal intensities (SI) were measured in the medial portion of the ACL and PCL, at the region of the inner meniscus and the adjacent proximal part of the popliteal muscle. To ensure consistency of ROI placement, all ROIs were placed by the same investigator.

\section{Qualitative Image Analysis}

Artifacts, image sharpness and homogeneity of fat suppression were rated in consensus by 3 investigators with more than 2 years of experience in musculoskeletal imaging. Image evaluations were done using a picture archiving and communication system (IMPAX 2.0, Agfa Healthcare, Bonn, Germany). Ratings were based on 5-point scales for:

- Artifacts (1: non-diagnostic; 2: poor=insufficient diagnostic confidence; 3 : moderate $=$ some artifacts, not interfering with di agnosis; 4 : good = minimal artifacts; 5 : excellent $=$ no artifacts),

- Image sharpness (1: non-diagnostic=blurry; 2: poor=knee structures can be identified, insufficient diagnostic confidence; 3: moderate $=$ sufficient for diagnosis, but low diagnostic confi dence; 4: good=diagnostic with high diagnostic confidence; 5: excellent $=$ crispy images ) and

- Homogeneity of fat suppression (1=non-diagnostic; 2 : poor= central and peripheral inhomogeneities; 3 : moderate $=$ major inhomogeneities (central); 4: good $=$ minor inhomogeneities (peripheral); 5: excellent $=$ no inhomogeneities).

The sum of the scores $(a+b+c)$ was used as a measure of the overall image quality ( $13-15$ points: excellent; $10-12$ points: good; $7-9$ points: moderate; $3-6$ points $=$ poor $)$.

\section{Diagnostic Accuracy}

In the 13 patients who underwent AKS, two radiologists with more than 10 years of experience in musculoskeletal imaging who were blinded to the histories of the patients and the results of AKS independently evaluated the presence or absence $(1 / 0)$ of the following lesions: ligamental tears and/or degeneration, meniscal tears and/ or degeneration, and cartilage lesions (grade $>2$ according to Outerbridge classification; medial/lateral femoral, medial/lateral tibial, retropatellar). Image evaluation was performed with time gaps of 4 weeks between 2D- and 3D-PDwFS readings in a randomized order. Readers were free to reconstruct any desired imaging plane using the integrated MPR software tool of the PACS.

\section{Statistical Analysis}

Statistical analysis was performed using SPSS (IBM SPSS Statistics 22.0, Armonk, New York). Data concerning CR measurements were presented as mean scores with standard deviations. To determine the statistical significance of CR measurements and of the qualitative ratings, Student's t-tests were performed. P-values lower than 0.05 were considered significant. Inter-observer agreement for diagnostic efficiency was estimated by Cohen's kappa statistics, where $\mathrm{K}=0.81-1.0$ was considered as almost perfect agreement, $\mathrm{K}=0.61-0.8$ as substantial agreement, $\mathrm{K}=0.41-0.6$ as moderate agreement, $\mathrm{K}=0.21-0.4$ as fair agreement, $\mathrm{K}=0-0.2$ as slight agreement and $\mathrm{K}<0.2$ as no agreement [15]. With AKS as the standard of reference, the sensitivity, specificity, accuracy, falsepositive and false-negative results of 2D- and 3D-PDwFS images for the detection of knee pathologies were calculated.

\section{Results \\ $\nabla$}

All examinations were successfully completed by the participants of the study.

\section{Image Contrast}

The results of CR measurements revealed higher contrast ratios of all investigated knee structures (ACL, PCL and MEN) in 3D-PDwFS compared to 2D-PDwFS ( $p<0.01$ for ACL/PCL, $p=0.07$ for MEN). These differences were most pronounced in the ACL. Image contrast was higher in all investigated structures in 3D-PDwFS. However, in 2D-PDwFS the contrast ratio of the ACL was lower than that of the PCL and MEN ( $\odot$ Table 2 ).

\section{Image Quality}

The overall image quality was rated significantly higher in 3DPDwFS compared to 2D-PDwFS with fewer artifacts and a higher homogeneity of fat suppression ( $\bullet$ Table 3 ). The most pronounced difference in image quality was observed when analyzing the presence of artifacts comparing sagittal 2D-PDwFS to sagittal MPR reconstructions of 3D-PDwFS. This was due to strong pulsation artifacts in the $2 \mathrm{D}$ technique in the phase encoding direction ( $\bullet$ Fig. 1 ).

Table 2 Measurements of contrast ratios (CR) in 2D-and 3D-PDwFS. The results of $C R$ measurement were higher for all investigated knee structures (ACL, PCL and MEN) in 3D-PDwFS compared to the $2 \mathrm{D}$ technique.

Tab. 2 Messungen des Kontrastverhältnisses (CR) in 2D- und 3D-PDwFS. Verglichen mit der 2D-PDwFS waren die CR-Werte für alle untersuchten Kniestrukturen (ACL, PCL und MEN) höher in der 3D-PDwFS.

\begin{tabular}{|lccc|} 
& 3D-PDwFS & 2D-PDwFS & p-value \\
\hline CR ACL/muscle & $0.62 \pm 0.24$ & $0.47 \pm 0.25$ & $<0.01$ \\
\hline CR PCL/muscle & $0.75 \pm 0.13$ & $0.68 \pm 0.15$ & $<0.01$ \\
\hline CR MEN/muscle & $0.73 \pm 0.11$ & $0.71 \pm 0.11$ & 0.07
\end{tabular}

CR: contrast ratio; $\mathrm{ACL}$ : anterior cruciate ligament; $\mathrm{PCL}$ : posterior cruciate ligament; MEN: meniscus; FS: fat suppression; PDwFS: proton density-weighted fat-suppressed sequence. 
Though significant, the homogeneity of fat suppression was only somewhat higher in 3D-PDwFS ( $\bullet$ Fig. 2). Image sharpness, on the other hand, was rated significantly higher in 2D-PDwFS ( $\bullet$ Table 3 ).

\section{Comparison to arthroscopic knee surgery}

In a subgroup of 13 patients who received AKS, 33/182 analyzed regions contained lesions. These included 6 meniscal and 6 ligamental tears, 16 cartilage lesions, 4 degenerative lesions of the meniscus and one giant cell tumor. The sensitivity, specificity, false-positive and false-negative results were similarly high for both readers when using 2D- vs. 3D-PDwFS ( $\bullet$ Table 4). Substantial inter-observer agreement for lesion detection was found for both readers using either 2D- or 3D-PDwFS (Cohen's kappa coefficients; 3D-PDwFS: 0.75; 2D-PDwFS: 0.62).

Both readers considered the possibility to reconstruct MPR in arbitrary planes out of isotropic 3D-PDwFS data sets advantageous as these allowed for better depiction of small and curved anatomic structures ( $\bullet$ Fig. 3 ) and lesions ( $\bullet$ Fig. 4 ).

Table 3 Parameters of image quality of 2D- and 3D-PDwFS. Overall image quality was higher in 3D-PDwFS with significantly fewer artifacts and (though less pronounced) higher homogeneity of fat suppression. Image sharpness was significantly higher in 2D-PDwFS.

Tab. 3 Parameter der Bildqualität der 2D- und 3D-PDwFS. Die Gesamtbildqualität war höher in der 3D-PDwFS, wobei sich signifikant weniger Artefakte und (etwas geringer ausgeprägt) eine homogenere Fettunterdrückung zeigten. Die Bildschärfe wurde in der 2D-PDwFS signifikant höher bewertet.

\begin{tabular}{|lrrr|}
\hline & 3D-PDwFS & 2D-PDwFS & p-value \\
\hline artifacts & $4.5 \pm 0.35$ & $3.3 \pm 0.17$ & $<0.01$ \\
\hline image sharpness & $2.6 \pm 0.23$ & $3.6 \pm 0.14$ & $<0.01$ \\
\hline homogeneity of FS & $3.7 \pm 0.21$ & $3.6 \pm 0.13$ & $<0.01$ \\
\hline overall image qualitative & $10.9 \pm 0.63$ & $10.5 \pm 0.28$ & $<0.01$
\end{tabular}

CR: contrast ratio; $\mathrm{ACL}$ : anterior cruciate ligament; $\mathrm{PCL}$ : posterior cruciate ligament; MEN: meniscus; FS: fat suppression; PDwFS: proton density-weighted fat-suppressed sequence.

\section{Discussion}

$\nabla$

Recent advances in MRI have led to the introduction of different $3 \mathrm{D}$ techniques aiming at improving image quality and diagnostic efficiency and facilitating sequence planning, whereas the routinely used 2D technique needs ideal planning of imaging planes in different orientations [3-6]. Early 3 D techniques applied gradient echo sequences, which not only suffered from long acquisition times, but also proved insufficient for accurate evaluation of ligaments, menisci and bone marrow changes. This was only later possible with the introduction of 3D-TSE techniques [7-9, 16, 17]. Further major improvements were achieved at higher field strengths and by using parallel imaging techniques and multichannel surface coils, which both reduced acquisition times and improved image quality [18-23]. This study shows the feasibility of a $3 \mathrm{D}$ proton-weighted fat-suppressed knee MRI sequence at 3.0T. The sequence is shown to provide sufficient image quality to replace multiplanar proton-weighted fat-suppressed $2 \mathrm{D}$ sequences in a reasonable scan time.

Both quantitative and qualitative parameters of image quality were equal or superior in 3D-PDwFS compared to 2D-PDwFS. Only in-plane image sharpness was rated superior in 2D-PDwFS. In a subset of patients, who also received both the study imaging protocol and AKS, the single 3D-PDwFS sequence and multiplanar 2D-PDwFS revealed similar diagnostic accuracy in the detection of various knee lesions.

The acquisition of a $3 \mathrm{D}$ data set allows for arbitrary multiplanar reformations without the need for additional sequences to obtain specific angulations that may be necessary to visualize small knee structures. $2 \mathrm{D}$ sequences are of limited value for multiplanar reformatting due to their inherently non-isotropic voxels resulting from thick slices, imaging gaps and partial-volume artifacts that may negatively affect image quality and obscure small pathologies $[5,7]$. In order to overcome these limitations and produce isotropic data sets, the slice thickness of 2D-PDwFS would have to be substantially decreased. However, thinner slices require long scan times and small voxel sizes lead to decreased signal-to-noise [18, 19]. $3 \mathrm{D}$ sequences are more suitable for the acquisition of small voxels due to their inherently higher signal level, but they require longer acquisition times than $2 \mathrm{D}$ sequences $[4,8,20,21]$.
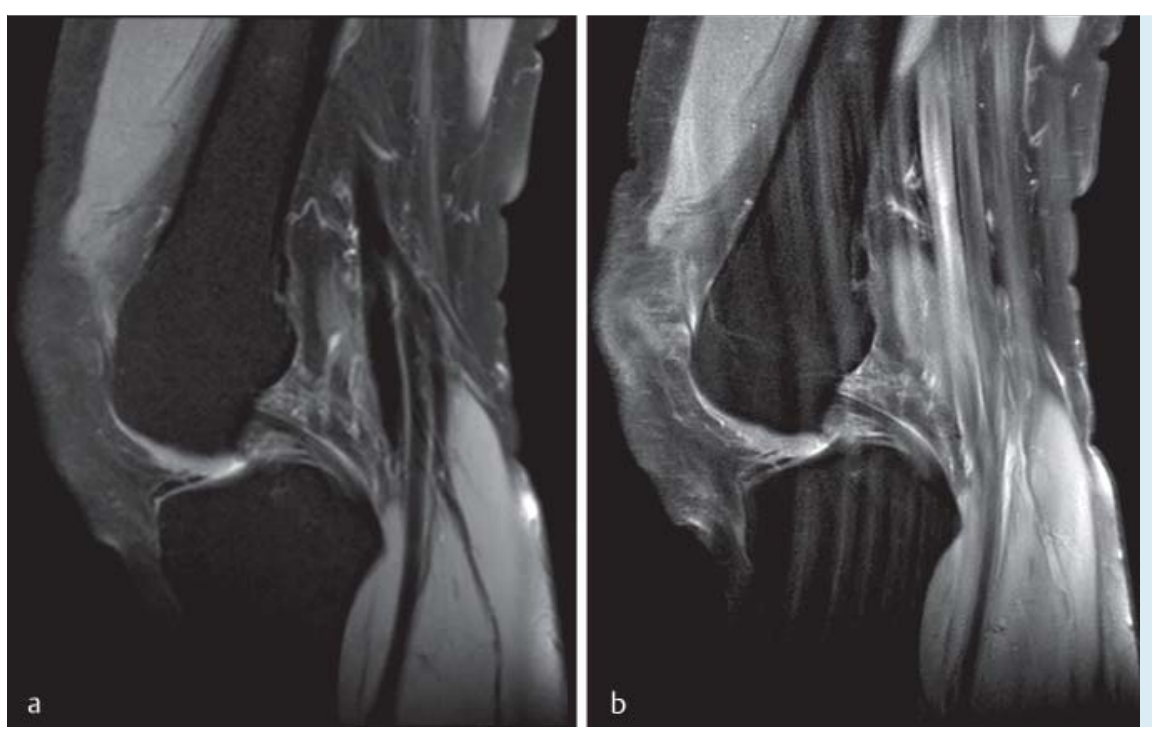

Fig. 1 Left knee of a 42-year-old female with anterolateral pain at the level of the patella. Note the lack of pulsation artifacts in the sagittal multiplanar reconstruction of the $3 \mathrm{D}$ proton density-weighted fatsuppressed image a compared to the $2 \mathrm{D}$ image $\mathbf{b}$.

Abb. 1 Linkes Knie einer 42 jährigen Frau mit Schmerzen anterolateral im Bereich der Patella. Es zeigen sich keine Pulsationsartefakte in der sagittalen multiplanaren Rekonstruktion der 3D-Proton-gewichteten fettunterdrückten Aufnahme a verglichen mit der 2D-Aufnahmein in Bild $\mathbf{b}$. 

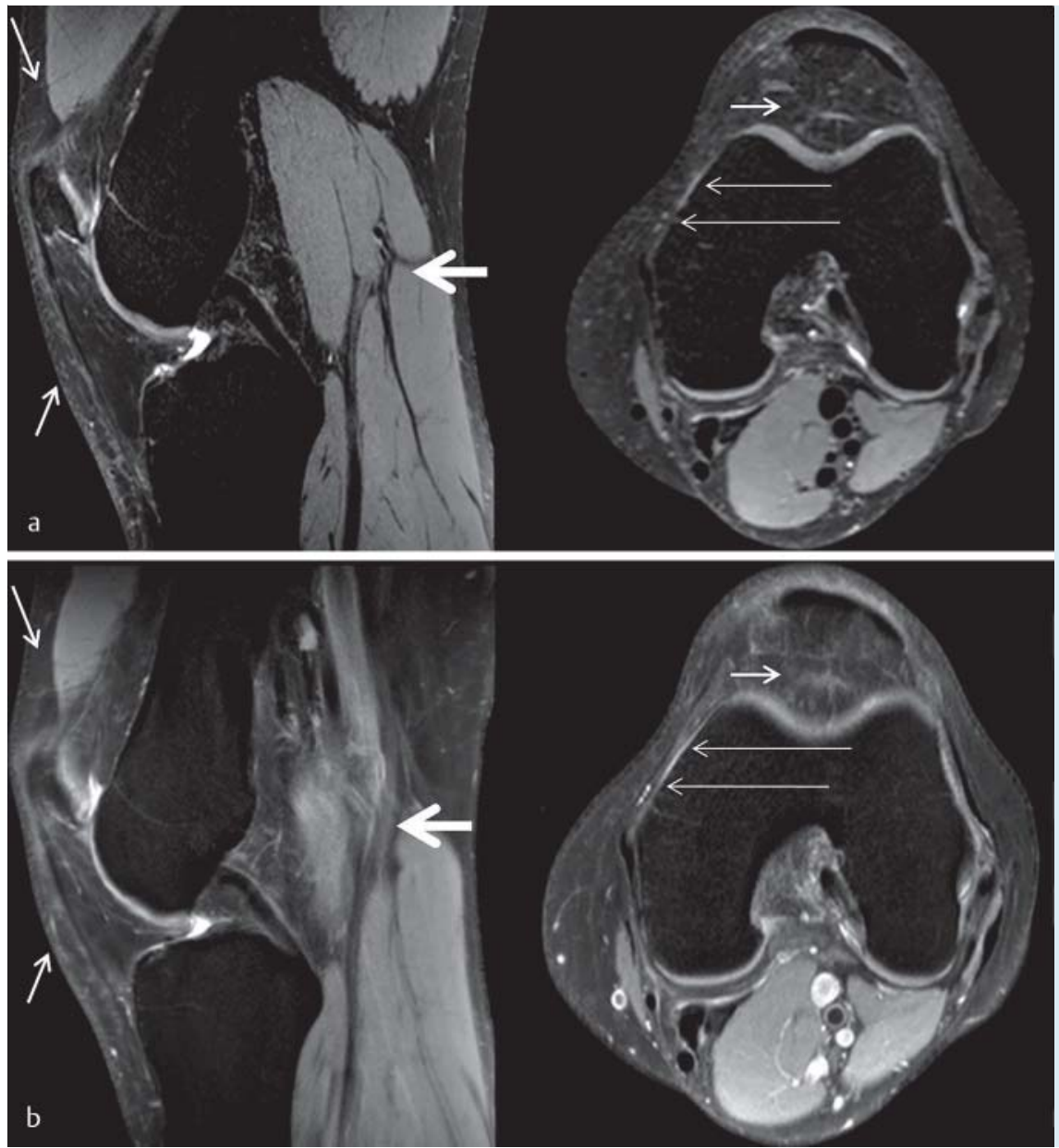

Fig. 2 Sagittal and transverse images in a 35-yearold man with pain of the right medial knee after a soccer game. Note the more homogeneous fat suppression (thin arrows), the reduction of artifacts (thick arrows) and the somewhat lower image sharpness (long arrows in axial slices) in a $3 \mathrm{D}$ proton density-weighted and fat-suppressed images (PDwFS) compared to $\mathbf{b}$ 2D-PDwFS.

Abb.2 Sagittale und transversal Aufnahmen des Knies eines 35 jährigem Mann bei medialen Schmerzen nach einem Fußballspiel. Die 3D-Proton-gewichteten fettunterdrückten Aufnahmen (PDwFS) a zeigen eine homogenere Fettunterdrückung (dünne Pfeile), geringere Artefakte (dicke Pfeile) und eine etwas niedrigere Bildschärfe (lange pfeile in den axialen Schichten) als die 2D-PDwFS b.
Table 4 Diagnostic accuracy of 2D- and 3D-PDwFS compared to arthroscopic knee surgery in 13 patients.

Tab. 4 Diagnostische Genauigkeit der 2D- und 3D-PDwFS verglichen zu Arthroskopien bei 13 Patienten.

\begin{tabular}{lllll} 
& reader 1 & \multicolumn{3}{c}{ reader 2 } \\
& 2D-PDwFS & 3D-PDwFS & 2D-PDwFS & 3D-PDwFS \\
\hline sensitivity & $81.8 \%$ & $81.8 \%$ & $81.8 \%$ & $78.8 \%$ \\
\hline specificity & $85.9 \%$ & $87.9 \%$ & $93.3 \%$ & $92.6 \%$ \\
\hline accuracy & $85.2 \%$ & $86.8 \%$ & $91.2 \%$ & $90.1 \%$ \\
\hline false-positive diagnoses & $21 / 149$ & $18 / 149$ & $10 / 149$ & $11 / 149$ \\
\hline - ligamental lesions & 2 & 1 & 1 & 0 \\
\hline - meniscal lesions & 5 & 5 & 3 & 4 \\
\hline - cartilage lesions & 3 & 4 & 2 & 0 \\
\hline - meniscal & 11 & 8 & 4 & 7 \\
\hline $\begin{array}{l}\text { fegeneration } \\
\text { false-negative }\end{array}$ & $6 / 33$ & $6 / 33$ & $6 / 33$ & $7 / 33$ \\
(missed lesions) & & & & \\
\hline - ligamental lesions & 0 & 0 & 0 & 0 \\
\hline - meniscal lesions & 0 & 0 & 2 & 2 \\
\hline - cartilage lesions & 5 & 6 & 4 & 5 \\
\hline - meniscal & 1 & 0 & 0 & 0 \\
\hline degeneration & & & & \\
\hline
\end{tabular}

PDwFS: proton density-weighted fat-suppressed sequence.
To shorten the scan time, 3D-PDwFS includes a modulation of flip angles by a refocusing radiofrequency angle technique, parallel imaging in both the phase- and slice-encoding direction and partial-Fourier acquisition [18-23]. A T/R-knee coil with high signal-to-noise and multiple elements was used at a field strength of 3.0 T to allow for the implementation of these techniques. The T/R-coil allows for a higher B1 amplitude due to the reduced excited field of view and for a reduced overall SAR compared to conventional techniques that use the body coil for excitation [24, 25]. The results are a higher signal-to-noise ratio, sharper pulse profiles and shorter pulse durations. The latter two points are particularly important for fast spin echo sequences with long echo trains where the total echo train length needs to be limited to avoid blurring, signal loss and loss of contrast. The large-slab excitation pulse of 3D-PDwFS allows for high signal-to-noise in spite of small voxel volumes, for a homogenous fat suppression ( $\bullet$ Fig. 2), for decreased pulsation artifacts, and for high contrasts in proton-weighted imaging.

The increased contrast of anatomic structures in 3D-PDwFS was most pronounced in the ACL. In general, differences in contrast parameters may be a result of excitation-related (e.g.; $2 \mathrm{D}$ vs. $3 \mathrm{D}$ techniques), sequence-related (e.g.; TR, TE, FA), and field strength-related (e.g.; $1.5 \mathrm{~T}, 3.0 \mathrm{~T}$ ) parameters [22 - 26].

Another mechanism for markedly lower contrast of the ACL in 2DPDwFS compared to 3D-PDwFS may be a partial volume effect that results from the small thickness of the ligament and its orientation in space. The latter is difficult to fully account for in sagittally oriented $2 \mathrm{D}$ slices and likely leads to partial volume effects that 


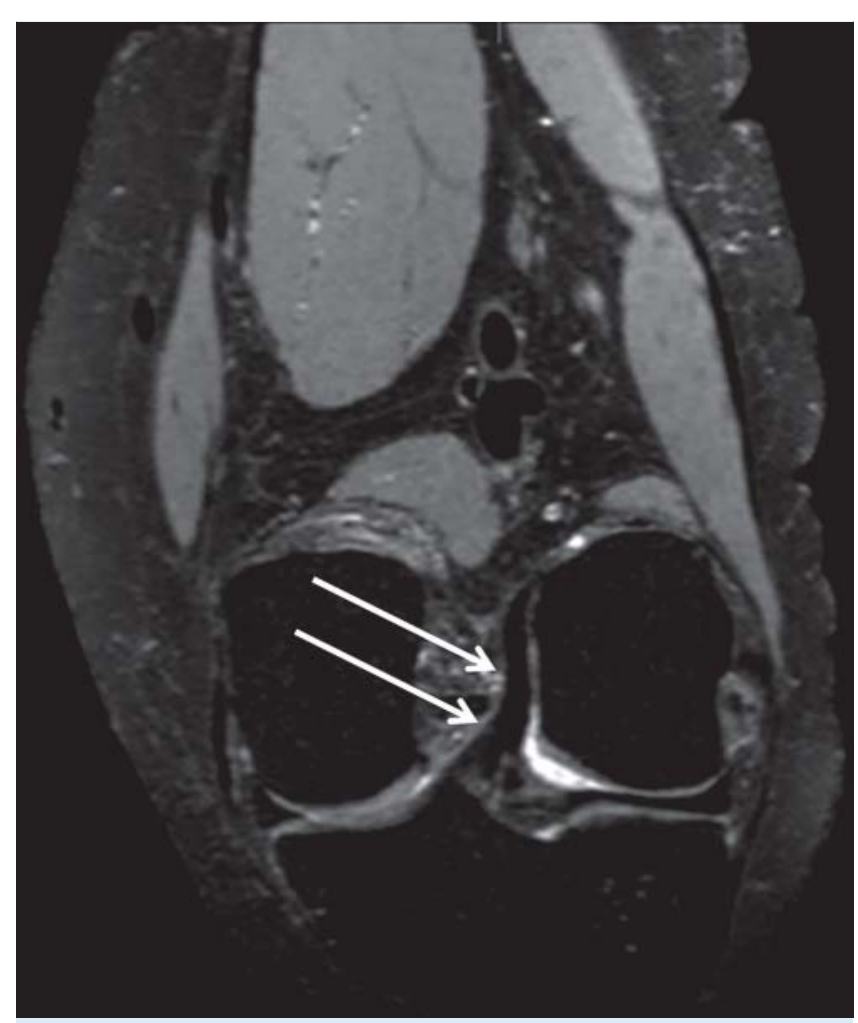

Fig. 3 Para-coronal MPR along the anterior cruciate ligament in 3D-PDwFS in a 34-year-old man.

Abb. 3 Para-koronare MPR entlang des vorderen Kreuzbandes in der 3D-PDwFS bei einem 34 jährigen.

lower the contrast of the ligament. Overall, the higher contrast of knee structures together with the better delineation of small pathologies, e.g. meniscal tear, in 3D-PDwFS may be regarded as an additional advantage of 3D-PDwFS. Similar observations were made in 3D-TSE intermediate-weighted sequences [5-7].

The significantly lower incidence of artifacts in 3D-PDwFS was mostly related to the decreased pulsation artifacts that are usually observed in 2D-PDwFS, especially in sagittal planes due to AP phase-encoding direction. These were not observed in sagittal MPR of 3D-PDwFS and may be attributed to the excitation pulse that covers a larger slab in 3D-PDwFS [5, 7, 24, 26, 27]. This has certain advantages in the case of preparation pulses, e.g. for fat suppression or inversion. Also the slice profile in $2 \mathrm{D}$ stretches over an entire slab in $3 \mathrm{D}$ and eliminates cross-talk between slices. Additional artifacts were not observed in 3D-PDwFS.

Image sharpness, on the other hand, was rated higher in 2DPDwFS than in MPR of 3D-PDwFS in corresponding planes. This may be attributed to a lower in-plane resolution of 3D-PDwFS compared to $2 \mathrm{D}$-PDwFS $(0.40 \times 0.44$ vs. $0.63 \times 0.63)$. However, the sensitivity, specificity and accuracy of 2D- and 3D-PDwFS in the subset of 13 patients who received AKS revealed no differences of the two sequences regarding lesion detection. As a result, the overall image quality of 3D-PDwFS was rated higher than that of 2D-PDwFS despite the lower in-plane resolution.

The availability of multiplanar reformats in any desired imaging plane in 3D-PDwFS was considered an advantage of 3D-PDwFS by both readers, particularly when analyzing oblique and curved structures including the cruciate ligaments. The acquisition of a complete $3 \mathrm{D}$ data set of the knee allows for reconstruction of arbitrary imaging planes as needed by the radiologist without acquiring additional carefully angulated sequences. Therefore, even though scan times of 3D-PDwFS and three standard planes of multiplanar 2D-PDwFS are in the same range (6:30 min. vs. $6: 40$ min.), the total scan time of knee examinations in clinical practice may benefit from the acquisition of a $3 \mathrm{D}$ data set. Another advantage of 3D-PDwFS may be reduced planning time of the MR examination for the MR technician due to the fact that careful angulations along small anatomic structures are not required when acquiring large $3 \mathrm{D}$ data sets $[28,29]$.

The fact that the assessment of the image quality including image sharpness, homogeneity of fat suppression and artifacts was performed in consensus reading instead of independent readers may be regarded as a limitation of this study. For instance, a recent study by Pass et al. observed reduced agreement between two independent observers for menisci and articular assessment using a $3 \mathrm{D}$ fast spin echo proton density fat-saturated technique compared to the standard $2 \mathrm{D}$ technique at 1.5 Tesla. However, the accuracy, sensitivity and specificity in the detection of knee pathologies were calculated using the standard $2 \mathrm{D}$ protocol as the reference standard in that particular study [30]. Our study involved both quantitative and qualitative parameters to analyze the performance of both techniques and arthroscopic surgery was taken as the reference standard whenever available in a subgroup of $25 \%$ of the patients. The small number of patients who received arthroscopy as a reference standard may be regarded as a limitation of the study and larger follow-up trials will be necessary to validate the preliminary results of the current study as it has been shown earlier regarding pathologies of the articular cartilage [1].

The non-randomized sequence order (first 2D-PDwFS, second 3D-PDwFS) may also be regarded as a limitation of this study. In patients who do not tolerate long acquisition procedures, motion artifacts may be more likely in the sequence acquired second. However, the overall image quality was rated higher in the sequence acquired second in this study, thus supporting the finding of the non-inferiority of 3D-PDwFS.

In 3D-PDwFS, temporary patient movement may affect the entire $3 \mathrm{D}$ data set and therefore all reconstructed planes, whereas in 2DPDwFS the same movement may only affect a single plane. In this case, repeating the 3D-PDwFS sequence is more time-consuming than repeating a single $2 \mathrm{D}-\mathrm{PDwFS}$ plane that was acquired during the short movement phase. In these particular patients, a prolongation of total scan time may be regarded as a possible drawback of the $3 \mathrm{D}$ technique. However, we did not observe any case with impaired diagnostic image quality due to movement artifacts in 52 consecutive patients covering a wide spectrum of knee diseases. Finally, the optimal trade-off between high spatial resolution, sufficient signal-to-noise and scan time was not systematically analyzed in this study and may be further optimized. However, we observed high diagnostic accuracy in a subgroup of patients who received AKS as the standard of reference procedure with good sensitivity, specificity and predictive values for both 3DPDwFS and 2D-PDwFS. Dedicated future studies will be required to investigate whether a further increase in diagnostic accuracy even beyond that of 2D-PDwFS may be possible using an optimized $3 \mathrm{D}$ proton density-weighted fat-suppressed sequence.

\section{Conclusion}

\section{$\nabla$}

The acquisition of a $3 \mathrm{D}$ proton density-weighted fat-suppressed data set with high spatial resolution can be realized in a reasonable scan time with high image quality at 3 Tesla. The sensitivity, speci- 

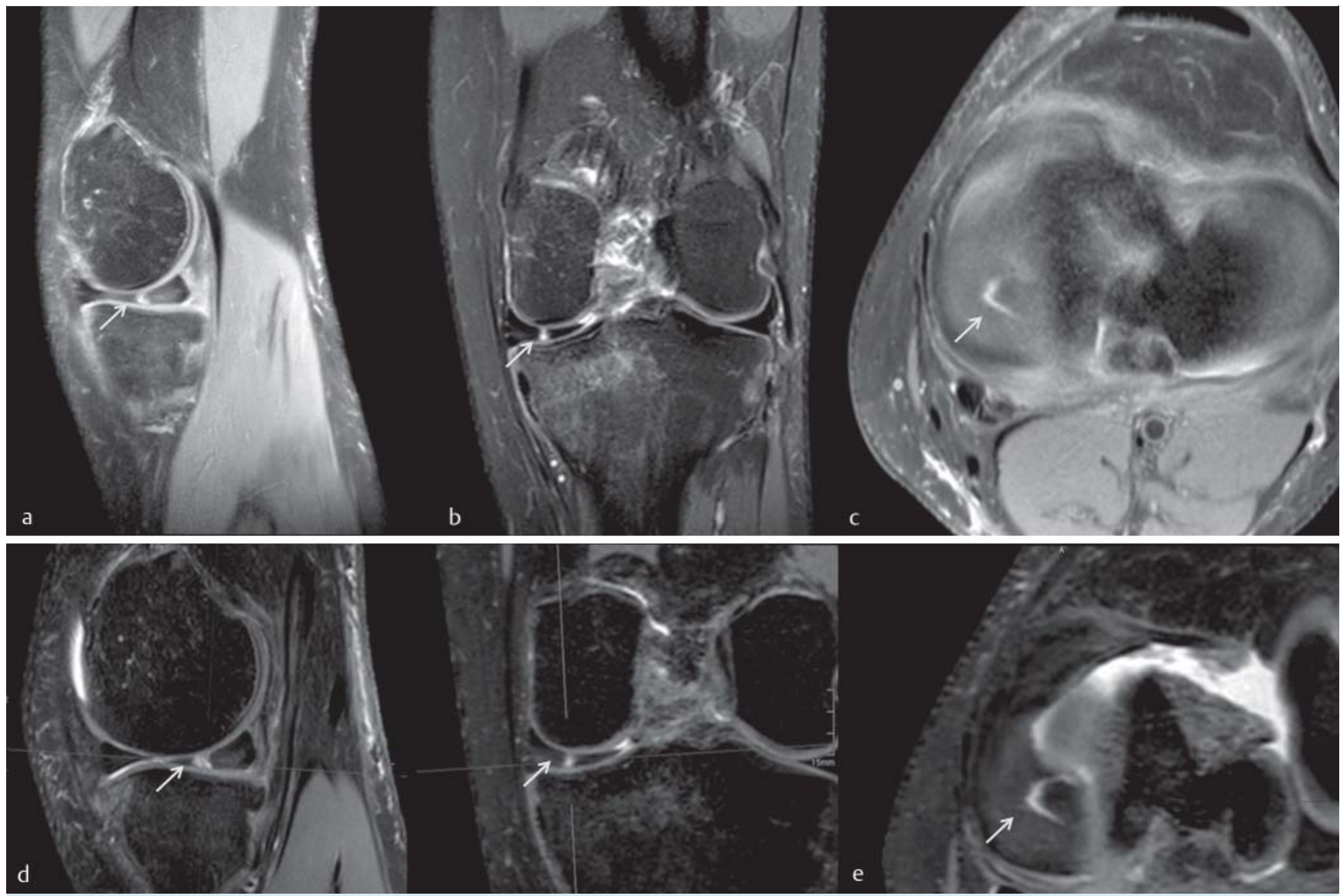

Fig. 4 Sagittal, coronal and transverse 2D-PDwFS knee images a-c showing a rupture of the posterior horn of the medial meniscus (arrows) in a 19-yearold male patient. Para-transverse multiplanar reformats along the axis of the posterior horn of the medial meniscus ( $\mathbf{d}$ = planning of optimal plane on a non-angulated coronal MPR of 3D-PDwFS, e = angulated multiplanar reconstruction of 3D-PDwFS) lead to optimized visualization of the lesion (arrows).
Abb.4 Sagittale, koronare und transversale 2D-PDwFS Knie-Aufnahmen a-c. Die Aufnahmen zeigen eine Ruptur des Innenmeniskushinterhorns (Pfeile) eines 19 jährigen Patienten. $\mathbf{d}$ = Planung der optimalen Ebene einer para-transversalen multiplanaren Reformation entlang der Achse des Innenmeniskushinterhorns anhand einer nicht-angulierten koronaren MPR der 3D-PDwFS. Die angulierte multiplanare Rekonstruktion der 3D-PDwFS e führt zu einer optimierten Visualisierung der Läsion (Pfeile). ficity and diagnostic accuracy were similar to that of standard multiplanar 2D proton density-weighted fat-suppressed images in a subset of 13 patients who received arthroscopic knee surgery. Post-processing by reconstruction of multiplanar reformats in arbitrary planes using isotropic voxels may serve as a diagnostic benefit of $3 \mathrm{D}$ data sets.

\section{Clinical relevance of the study}

- Multiplanar 2 D imaging is the current standard in knee MRI but suffers from disadvantages such as non-isotropic voxels, thick sections and imaging gaps.

- Separate sequences for each perpendicular plane may be time-consuming especially when additional regions of interest have to be studied in more detail.

$3 \mathrm{D}$ volume data sets with isotropic voxels can be acquired in a reasonable scan time at 3.0 T with high image quality and allow for multiplanar reformation.

\section{References}

1 Gustas CN, Blankenbaker DG, Rio AM et al. Evaluation of the Articular Cartilage of the Knee Joint Using an Isotropic Resolution 3D Fast SpinEcho Sequence With Conventional and Radial Reformatted Images. Am J Roentgenol 2015; 205: 371-379

2 Van Dyck P, Vanhevel F, Vanhoenacker FM et al. Morphological MR imaging of the articular cartilage of the knee at $3 \mathrm{~T}$-comparison of standard and novel 3D sequences. Insights Imaging 2015; 6: 285-293

3 Kijowski R, Blankenbaker DG, Klaers JL et al. Vastly undersampled isotropic projection steady-state free precession imaging of the knee: diagnostic performance compared with conventional MR. Radiology 2009; 251: $185-194$

4 Wieslander SB, Rappeport ED, Lausten GS et al. Multiplanar reconstruction in MR imaging of the knee. Comparison with standard sagittal and coronal images. Acta Radiol 1998; 39: 116-119

5 Gold GE, Busse RF, Beehler C et al. Isotropic MRI of the knee with 3D fast spin-echo extended echo-train acquisition (XETA): initial experience. Am J Roentgenol 2007; 188: 1287 - 1293

6 Notohamiprodjo M, Horng A, Pietschmann MF et al. MRI of the knee at 3T: first clinical results with an isotropic PDfs-weighted 3D-TSE-sequence. Invest Radiol 2009; 44: 585-597

7 Kijowski R, Davis KW, Woods MA et al. Knee joint: comprehensive assessment with 3D isotropic resolution fast spin-echo MR imagingdiagnostic performance compared with that of conventional MR imaging at 3.0 T. Radiology 2009; 252: 486-495

8 Kijowski R, Gold GE. Routine 3D magnetic resonance imaging of joints. J Magn Reson Imaging 2011; 33: 758 - 771 
9 Ristow O, Steinbach L, Sabo G et al. Isotropic 3D fast spin-echo imaging versus standard $2 \mathrm{D}$ imaging at $3.0 \mathrm{~T}$ of the knee-image quality and diagnostic performance. Eur Radiol 2009; 19: 1263 -1272

10 Fellner C, Geissler A, Held P et al. Signal, contrast, and resolution in optimized PD- and T2-weighted turbo SE images of the knee. Journal of computer assisted tomography 1995; 19: 96-105

11 Schafer FK, Schafer PJ, Brossmann J et al. Value of fat-suppressed protondensity-weighted turbo spin-echo sequences in detecting meniscal lesions: comparison with arthroscopy. Acta Radiol 2006; 47: 385 - 390

12 Sonin AH, Pensy RA, Mulligan ME et al. Grading articular cartilage of the knee using fast spin-echo proton density-weighted MR imaging without fat suppression. Am J Roentgenol 2002; 179: 1159-1166

13 Schaefer FK, Schaefer PJ, Brossmann J et al. Value of fat-suppressed PDweighted TSE-sequences for detection of anterior and posterior cruciate ligament lesions-comparison to arthroscopy. Eur J Radiol 2006; 58: $411-415$

14 Reed ME, Villacis DC, Hatch GF 3rd et al. 3.0-Tesla MRI and arthroscopy for assessment of knee articular cartilage lesions. Orthopedics 2013; 36: e1060-e1064

15 Viera AJ, Garrett JM. Understanding interobserver agreement: the kappa statistic. Fam Med 2005; 37: 360 - 363

16 Crema MD, Roemer FW, Marra MD et al. Articular cartilage in the knee: current MR imaging techniques and applications in clinical practice and research. Radiographics 2011; 31: 37-61

17 Chagas-Neto FA, Nogueira-Barbosa MH, Lorenzato MM et al. Diagnostic performance of 3D TSE MRI versus 2D TSE MRI of the knee at $1.5 \mathrm{~T}$, with prompt arthroscopic correlation, in the detection of meniscal and cruciate ligament tears. Radiol Bras 2016; 49: 69-74

$18 \mathrm{Ha}$ TP, Li KC, Beaulieu CF et al. Anterior cruciate ligament injury: fast spin-echo MR imaging with arthroscopic correlation in 217 examinations. Am J Roentgenol 1998; 170: 1215 - 1219

19 Rajeswaran G, Lee JC, Healy JC. MRI of the popliteofibular ligament: isotropic 3D WE-DESS versus coronal oblique fat-suppressed T2W MRI. Skeletal Radiol 2007; 36: 1141 - 1146

20 Jung JY, Yoon YC, Jung JY et al. Qualitative and quantitative assessment of wrist MRI at 3.0T: comparison between isotropic 3D turbo spin echo and isotropic 3D fast field echo and 2D turbo spin echo. Acta Radiol 2013; 54: $284-291$

21 Gay SB, Chen NC, Burch JJ et al. Multiplanar reconstruction in magnetic resonance evaluation of the knee. Comparison with film magnetic resonance interpretation. Invest Radiol 1993; 28: 142 - 145

22 Busse RF, Brau AC, Vu A et al. Effects of refocusing flip angle modulation and view ordering in 3D fast spin echo. Magn Reson Med 2008; 60: 640-649

23 Busse RF, Hariharan $\mathrm{H}, \mathrm{Vu} \mathrm{A}$ et al. Fast spin echo sequences with very long echo trains: design of variable refocusing flip angle schedules and generation of clinical T2 contrast. Magn Reson Med 2006; 55: $1030-1037$

24 Bachschmidt TJ, Sutter R, Jakob PM et al. Knee implant imaging at $3 \mathrm{Te}-$ sla using high-bandwidth radiofrequency pulses. J Magn Reson Imaging 2015; 41: 1570 - 1580

25 Cao Z, ParkJ, Cho ZH et al. Numerical evaluation of image homogeneity, signal-to-noise ratio, and specific absorption rate for human brain imaging at $1.5,3,7,10.5$, and $14 \mathrm{~T}$ in an 8-channel transmit/receive array. J Magn Reson Imaging 2015; 41: 1432 - 1439

26 Li CQ Chen W, Rosenberg JK et al. Optimizing isotropic three-dimensional fast spin-echo methods for imaging the knee. J Magn Reson Imaging 2014; 39: 1417 - 1425

27 Gold GE, Reeder SB, Yu $\mathrm{H}$ et al. Articular cartilage of the knee: rapid three-dimensional MR imaging at 3.0 T with IDEAL balanced steadystate free precession-initial experience. Radiology 2006; 240: 546 - 551

28 Young S, Bystrov D, Netsch $T$ et al. Robust Anatomy Recognition for Automated MR Neuro Scan Planning. Proceedings of ISMRM 14th Scientific Meeting. Seattle, Washington: 2006; DOI: 1588

29 Springorum R, Hoogenraad F, Bergmans R et al. Automated versus Manual Scan Positioning: A Quantitative Analysis. Proceedings of ISMRM 14th Scientific Meeting. Seattle, Washington: 2006; DOI: 1593

30 Pass B, Robinson P, Hodgson $R$ et al. Can a single isotropic 3D fast spin echo sequence replace three-plane standard proton density fat-saturated knee MRI at 1.5 T? Br J Radiol 2015; 88: 20150189 